九州大学学術情報リポジトリ

Kyushu University Institutional Repository

\title{
A List of Dragonflies Collected in Bangladesh
}

Nomura, Shi ihe i

Alam, Md. Zinnatul

https://doi.org/10.5109/2588

出版情報: ESAKIA. 35, pp. 135-140，1995-01-31. Entomological Laboratory，Faculty of Agriculture, Kyushu University バージョン :

権利関係 : 


\title{
A List of Dragonflies Collected in Bangladesh')
}

\author{
Shiihei NOMURA \\ Entomological Laboratory, Faculty of Agriculture \\ Kyushu University, Fukuoka, 812 Japan \\ and \\ Md. Zinnatul ALAM \\ Entomology Department, Institute of Postgraduate Study in Agriculture \\ Salna, Gazipur, Bangladesh
}

\begin{abstract}
Seventeen species of dragonflies collected from Bangladesh are listed.
The dragonflies collected from IPSA and the other points in Bangladesh are identified and listed below. In this list, the collecting sites of some institutes are abbreviated as follows: IPSA (Institute of Postgraduate Study in Agriculture, Gazipur Dist.); MRS, Nawabganj (Mango Research Station, Chapai Nawabganj, Nawabganj Dist.); SRTI, Ishurdi(Sugarcane Research and Training Institute, Ishurdi, Pabna Dist.).
\end{abstract}

\section{Family Agrionidae}

\section{Ceriagrion coromandeliunum (Fabricius) [Indo-ki-ito-tonbo]}

Specimens examined. 1 male, 1 female, IPSA, 2. xi. 1993, S. Nomura leg.; 1 male, 1 female, SRTI, Ishurdi, 13-16. xi. 1993, S. Nomura leg.; 1 male, 2 females, Botanical Garden, Dhaka C., 13. ii. 1993, 0. Tadauchi leg.

2. Ischnura aurora aurora (Brauer) [Kibara-ito-tonbo]

Specimens examined. 3 males, IPSA, 1. xi. 1993, S. Nomura leg.; 4 males, 1 female, IPSA, 2. xi. 1993, S. Nomura leg..

Distribution. Oriental and Australian Regions.

1) Contribution from the Entomological Laboratory, Faculty of Agriculture, Kyushu University, Fukuoka (Ser. 4, No. 83). 


\section{Agriocnemis pygmaea (Rambur) [Hime-ito-tonbo]}

Specimens examined. 2 males, 4 females, IPSA, 1. xi. 1993, S. Nomura leg.; 11 males, 5 females, IPSA, 2. xi. 1993, S. Nomura leg.

Distribution. Oriental and Australian Regions.

4. Pseudagrion microcephalum (Rambur) [Ao-naga-ito-tonbo]

Specimens. examined. 1 male, 1 female, Balda Garden, Dhaka C., 29. x. 1993, S. Nomura leg.

Distribution. Oriental and Australian Regions.

\section{Pseudagrion laidlawi Fraser}

Specimens examined. 1 male, 1 female, IPSA, 1. xi. 1993, S. Nomura leg.; 1 female, IPSA, 2. xi. 1993, S. Nomura leg.

\section{Family Libelluridae}

\section{Diplacodes trivialis (Rambur) [Hime-tonbo]}

Specimens examined. 1 female, IPSA, 4. xi. 1993, S. Nomura leg.; 1 male, 2 females, IPSA, 2. xii. 1994, S. Nomura leg.; 1 female, SRTI, Ishurdi, 13-16. xi. 1993, S. Nomura leg.; 1 female, Bhowal forest, Gazipur Dist., 28. xi. 1993, S. Nomura leg..

Distribution. Oriental Region, China, India, Bangladesh.

\section{Bruchythemis contaminata (Fabricius) [Hime-ki-tonbo]}

Specimens examined. 1 female, IPSA, 4. xi. 1993, S. Nomura leg.; 2 females, IPSA, 2. xii. 1994, S. Nomura leg.

Distribution. Southeast Asia, India, Bangladesh.

8. Crocothemis servilia servilia (Drury) [Syoujou-tonbo]

Specimens examined. 1 female, IPSA, 4. xi. 1993, S. Nomura leg.; 1 male, 1 female, IPSA, 2. xii. 1994, S. Nomura leg.; 1 male, SRTI, Ishurdi, 13-16. xi. 1993, S. Nomura leg..

Distribution. Japan, Taiwan, China, Southeast Asia, India, Bangladesh.

9. Orthetrum pruinosum neglectum (Rambur) [Kofuki-syoujou-tonbo]

Specimens examined. 3 males, Satchari forest, Hobiganj Dist., 8-10. xi. 1993, Md. Z. Alam \& S. Nomura leg.

Distribution. Japan (Ryukyu), Taiwan, China, Indo-china, Tibet, Burma, India, Sri Lanka, Bangladesh.

\section{Orthetrum sabina sabina (Drury) [Haraboso-tonbo]}

Specimens examined. 2 females, Satchari forest, Hobiganj Dist., 8-10. xi. 1993, Md. Z. Alam \& S. Nomura leg.; 1 male, Bhowal forest, Gazipur Dist., 28. xi. 1993, S. Nomura leg.; 1 male, 1 female, Jaintapur, Sylhet Dist., 9. xi. 1993, Md. Z. Alam leg.; 1 male, Balda Garden, Dhaka C., 29. x. 1993, S. Nomura leg. 
Distribution. Oriental, Australian and Ethiopian Regions.

\section{Cratilla lineata (Brauer) [Usuguro-tonbo]}

Specimens examined. 1 male, 1 female, MRS, Nawabganj, 15. xi. 1993, S. Nomura leg.; 1 female, SRTI, Ishurdi, 13-16. xi. 1993, S. Nomura leg.; 2 females, Bhowal forest, Gazipur Dist., 28. xi. 1993, S. Nomura leg.

\section{Tholymis tillarga (Fabricius) [Ame-iro-tonbo]}

Specimens examined. 1 male, 1 female, Balda Garden, Dhaka C., 29. x. 1993, S. Nomura leg.; 1 male, 2 females, Bhowal forest, Gazipur Dist., 28. xi. 1993, S. Nomura leg..

Distribution. Oriental, Australian and Ethiopian Regions.

\section{Tremea basilaris burmeisteri Kirby}

Specimens examined. 1 male, 1 female, MRS, Nawabganj, 15. xi. 1993, S. Nomura leg.; 1 female, SRTI, Ishurdi, 13-16. xi. 1993, Md. Z. Alam leg.

\section{Trithemis pallidinervis (Kirby) [Ashi-naga-tonbo]}

Specimens examined. 1 male, 2 females, IPSA, 2. xi. 1993, S. Nomura leg.

\section{Neurothemis fulvia (Drury) [Aka-bekkou-tonbo]}

Specimens examined. 2 males, 1 female, Jaintapur, Sylhet Dist., 9. xi. 1993, S. Nomura leg.; 1 female, Satchari forest, Hobiganj Dist., 8-10. xi. 1993, S. Nomura leg.; 1 male, Madhupur, Tangail Dist., 22. xi. 1993, S. Nomura leg..

\section{Neurothemis tullia tullia (Drury) [Neguro-tonbo]}

Specimens examined. 3 females, IPSA, 1. xi. 1993, S. Nomura leg.; 2 males, IPSA, 2. xi. 1993, S. Nomura leg.; 2 males, IPSA, 2. xii. 1993, S. Nomura leg.; 6 males, SRTI, Ishurdi, 1316. xi. 1993, S. Nomura leg.; 1 female, Balda Garden, Dhaka C., 29. x. 1993, S. Nomura leg..

\section{Neurothemis intermedia atalanta Ris}

Specimens examined. 1 male, IPSA, 1. xi. 1993, S. Nomura leg.; 4 males, 1 female, Madhupur, Tangail Dist., 22. xi. 1993, S. Nomura leg.; 1 female, Bhowal forest, Gazipur Dist., 28. xi. 1993, S. Nomura leg.; 1 male, Satchari forest, Hobiganj Dist., 8-10. xi. 1993, S. Nomura leg.

\section{Acknowledgement}

We wish to express our special thanks to Dr. Syojiro Asahina (Tokyo Pref.) for his kind identification of materials and invaluable advices. Our cordial thanks are due to Prof. Katsura Morimoto and Associate Prof. Osamu Tadauchi of the Entomological Laboratory, Kyushu University for their continuous guidance and kind offer of the materials. We are also indebted to Dr. Kazuo Ogata (Kyushu Univ.) and Mr. Fazlar Rahman (IPSA) for their kind assistance in various ways. 


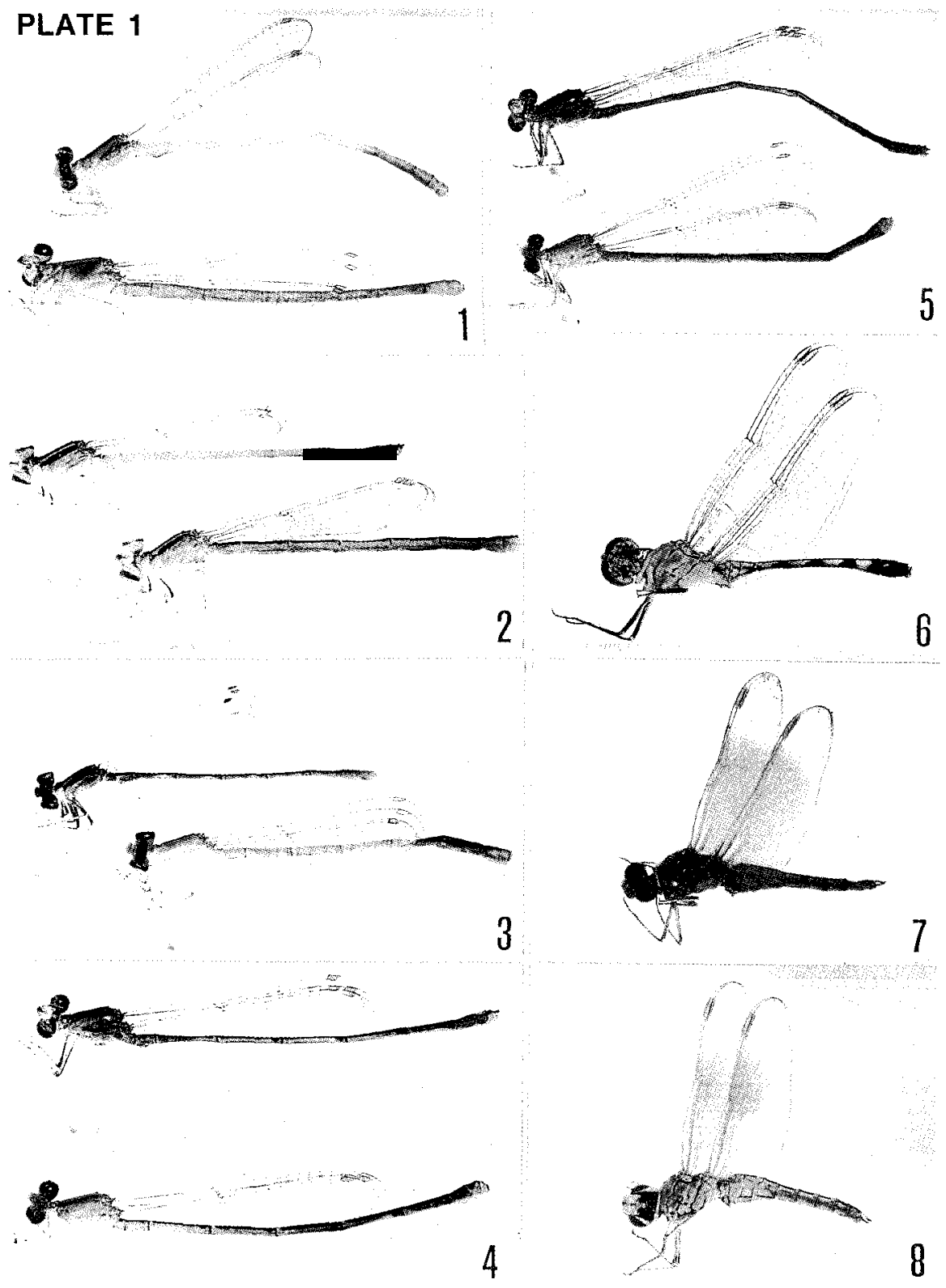

1. Ceriagrion coromandelianum (Fabricius), male and female; 2. Ischnura aurora aurora (Brauer), male and female; 3. Agriocnemis pygmaea (Rambur), male and female; 4. Pseudagrion microcephalum (Rambur), male and female; 5. Pseudagrion laidlawi Fraser, male and female, ; 6. Diplacodes trivialis (Rambur), male; 7. Brachythemis contaminata (Fabricius), male; 8. ditto, female. 


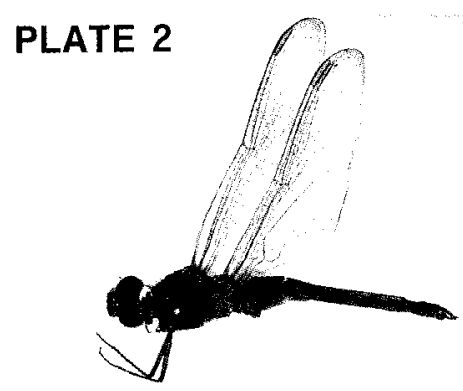

9
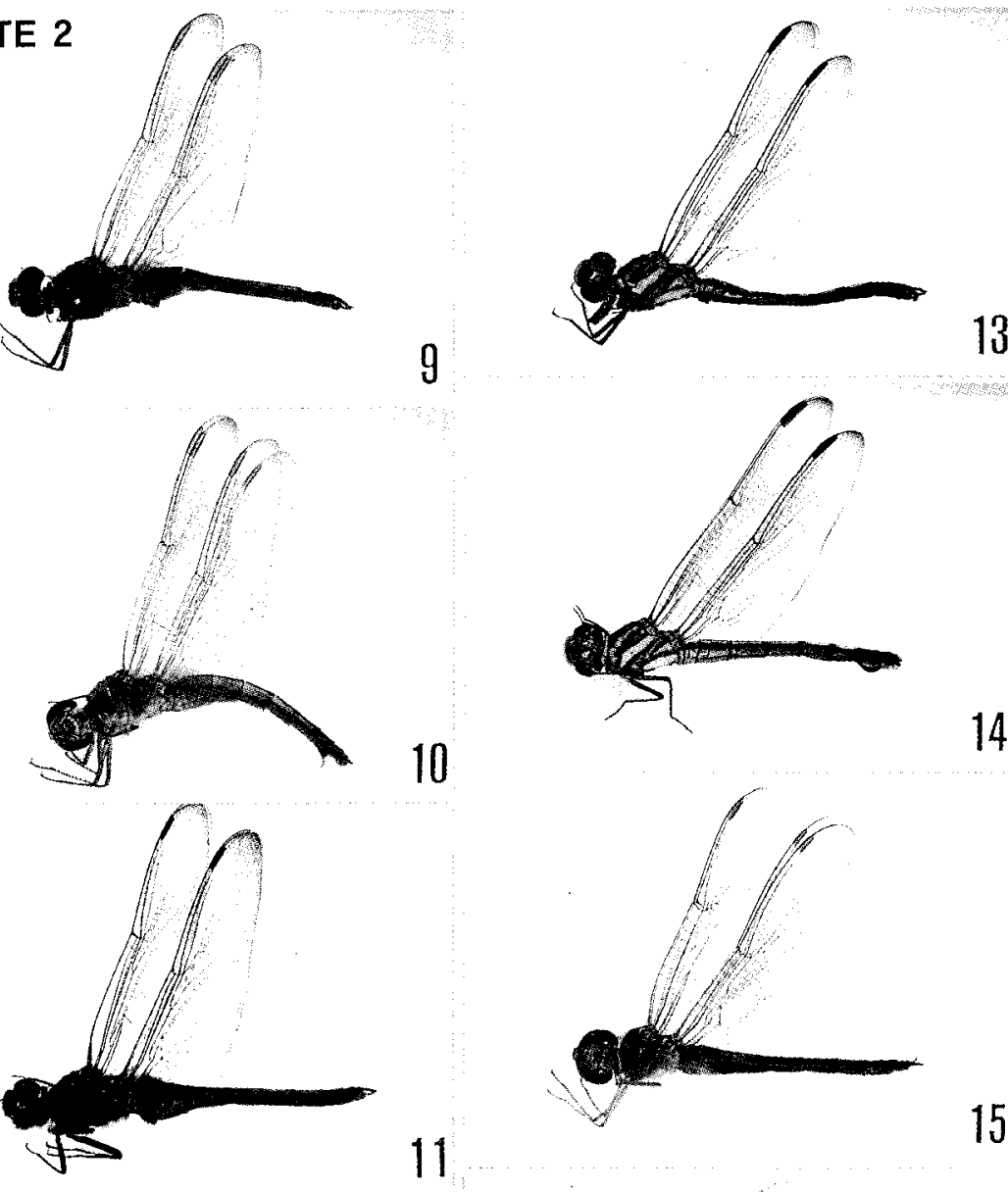

13

10
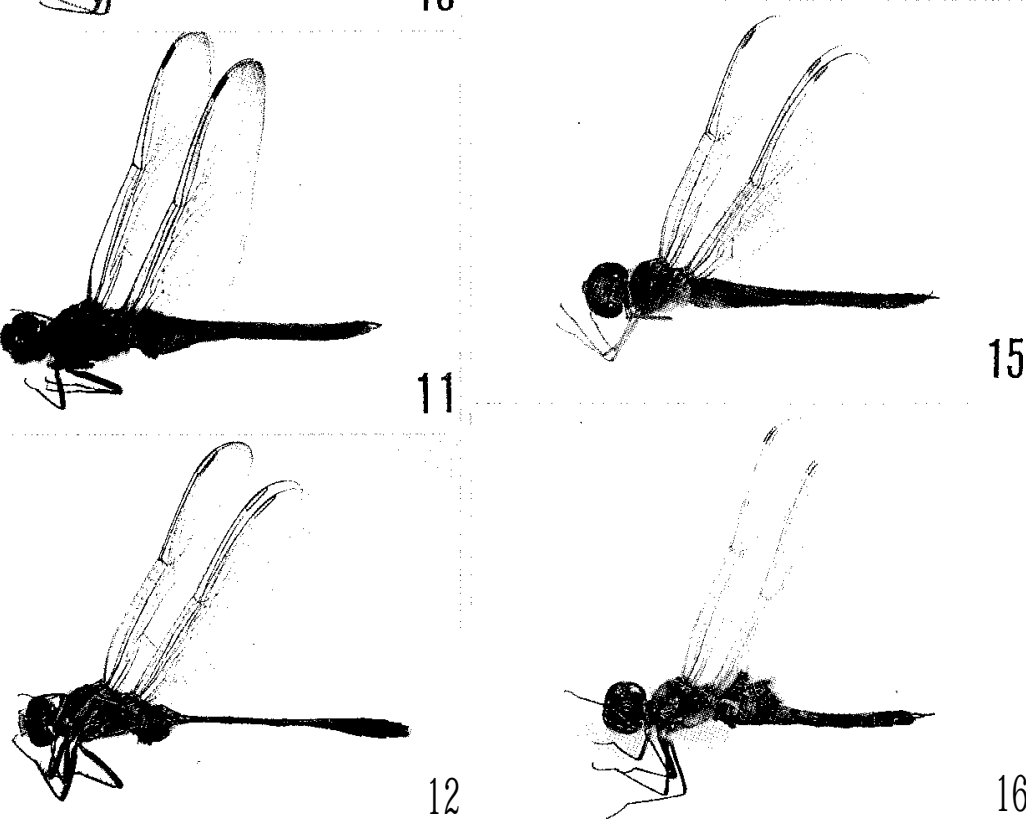

9. Crocothemis servilia servilia (Drury), male;10. ditto, female; 11. Orthetrum pruinosum neglectum (Rambur), male; 12, Orthetrum sabina sabina (Drury), male; 13. Cratilla lineata (Brauer), male; 14. ditto, female; 1.5. Tholymis tillarga (Fabricius), male; 16 Tremea basiluris burmeisteri Kirby. 


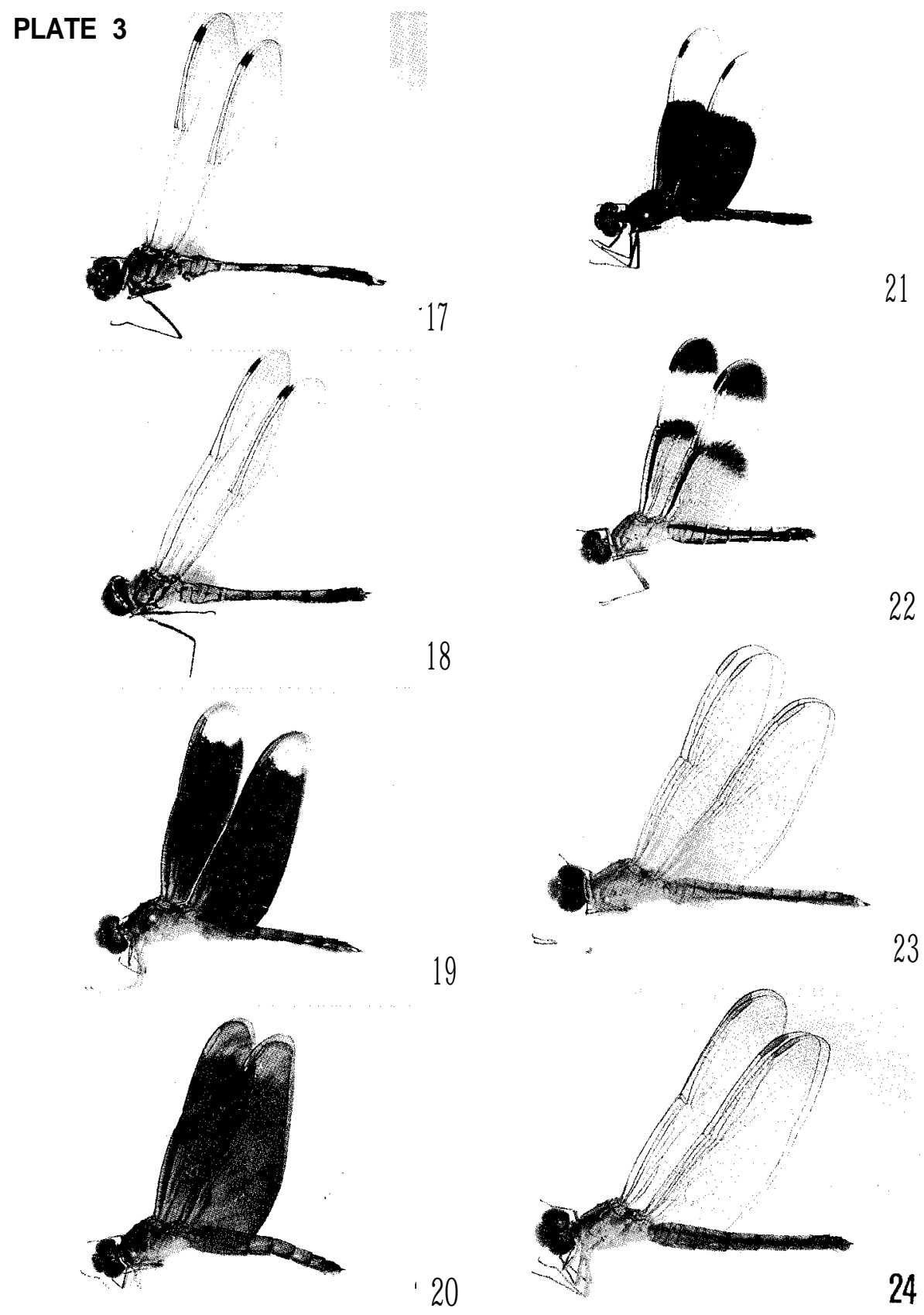

17. Trithemis pallidinervis (Kirby), male; 18. ditto, female; 19. Neurothemis fulvia (Drury), male; 20. ditto, female; 21. Neurothemis tullia tullia (Drury), male; 22. ditto, female; 23. Neurothemis intermedia atalanta Ris, male; 24. ditto, female. 